\title{
OPEN The prevalence of sleep disturbance among asthmatic patients in a tertiary care center
}

Tammam M. Alanazi ${ }^{1}$, Hazim S. Alghamdi ${ }^{1}$, Meshal S. Alberreet ${ }^{1}$, Abdulaziz M. Alkewaibeen ${ }^{1}$, Abdulrahman M. Alkhalefah ${ }^{1}$, Aamir Omair ${ }^{1,4}$, Hamdan AL-Jahdali ${ }^{1,2,3}$ \& Abdullah AL-Harbi ${ }^{1,2,3 凶}$

Sleep disturbances are commonly reported by patients with asthma. However, the prevalence of sleep disturbance and its association with the level of asthma control is unknown. The primary objective was to determine the prevalence of sleep disturbance among Saudi adult asthmatic patients attending pulmonary clinics at King Abdulaziz Medical City (KAMC). The study also aimed to compare sleep quality between controlled and uncontrolled asthma patients. The study was carried out in the outpatient pulmonary clinics at KAMC and utilized a cross-sectional survey. The survey included five different questionnaires: asthma control test and questionnaires related to the quality of sleep (Pittsburgh sleep quality index [PSOI], Epworth sleepiness scale [ESS], Berlin questionnaire [a measure of obstructive sleep apnea risk], and insomnia severity index [ISI]). Among the 200 asthma patients, $66 \%$ suffered from poor sleep quality (PSOI > 5), $43 \%$ were at high risk for obstructive sleep apnea, $25 \%$ had excessive daytime sleepiness (ESS $>10$ ), and $46.5 \%$ had significant clinical insomnia (ISI $\geq 10)$. Poor sleep quality was less common in patients with well-controlled asthma (37\%) compared to those with partially controlled asthma $(78 \%)$ and uncontrolled asthma $(82 \%)(p<0.001)$. Poor sleep quality was common among patients with asthma, particularly those with suboptimal levels of asthma control. Further studies are needed to better understand the interaction between these two conditions.

Asthma is a common chronic disease characterized by recurrent symptoms, variable airflow obstruction, bronchial hyper-responsiveness (BHR), and underlying inflammatory changes affecting mainly the airways ${ }^{1,2}$. It is estimated approximately 300 million people suffering from asthma worldwide ${ }^{2}$. A recent meta-analysis estimated pooled weighted prevalence rate of asthma is $14.3 \%$ among Saudi population ${ }^{3}$.

Sleep disorders are characterized by the inability to maintain good quality sleep or problems with the duration of sleep, which can have an impact on daytime activities. Examples of sleep disorders include obstructive sleep apnea (OSA), insomnia, difficulty maintaining sleep, and restless leg syndrome. Sleep disturbances are common complaints among asthma patients and can be manifested as difficulties initiating and maintaining sleep, as well as early morning awakenings ${ }^{4-7}$. Sleep disturbance at night may be partly due to poor asthma control and can also be related to diurnal variations in airflow limitation, with an increase in BHR and airway resistance at night ${ }^{8-10}$.

Other common conditions, such as gastroesophageal reflux disease (GERD), OSA, chronic rhinitis, and depression, are associated with asthma and can also lead to sleep disturbances and poor sleep quality independent of asthma control ${ }^{8,11-13}$. In addition, asthma is associated with a higher prevalence of OSA, especially in patients with difficult-to-control asthma ${ }^{14-16}$. A recent meta-analysis showed that the prevalence of OSA in adult asthma patients is estimated to be $50 \%$, with an odds ratio of 2.64 for OSA in asthma patients compared with non-asthma patients ${ }^{15}$. Poor sleep quality was associated with worse asthma control and quality of life, even after controlling for GERD and other covariates ${ }^{16}$.

Insomnia is also common among asthma patients. Thirty-seven percent of asthma patients have clinically significant insomnia, which has been associated with worse asthma symptoms, poor quality of life, and increased

${ }^{1}$ College of Medicine, King Saud Bin Abdulaziz University for Health Sciences (KSAU-HS), Riyadh, Saudi Arabia. ${ }^{2}$ Pulmonary Division, Department of Medicine, Ministry of National Guard-Health Affairs, King Abdulaziz Medical City, MC 1443, P.O. Box 22490, Riyadh 11426, Saudi Arabia. ${ }^{3}$ King Abdullah International Medical Research Center, Riyadh, Saudi Arabia. ${ }^{4}$ Department of Medical Education, College of Medicine, King Saud Bin Abdulaziz University for Health Sciences, Riyadh, Saudi Arabia. ${ }^{\circledR}$ email: harbia7@ngha.med.sa 
asthma-related health care utilization in the preceding year ${ }^{17}$. Furthermore, daytime sleepiness from sleep disturbance has been shown to affect $30-50 \%$ of asthma patients ${ }^{5,18}$.

The association between asthma severity and sleep disturbance is quite complex and needs to be addressed. The purpose of this study was to determine the prevalence of sleep disturbance among a sample of Saudi adult asthmatic patients. We also aimed to compare sleep quality between controlled and uncontrolled asthma patients.

\section{Materials and methods}

This cross-sectional study was conducted in the outpatient general pulmonary clinics at King Abdulaziz Medical City (KAMC) in Riyadh, Saudi Arabia. The study was approved by the Institutional Review Board Committee at King Abdullah International Medical Research Center. All patients who had a diagnosis of asthma and were scheduled to visit the pulmonary clinics from August 2018 to April 2019 were screened for eligibility. The inclusion criteria were the following; (1) ages 15 years and older; and (2) confirmed cases of bronchial asthma based on previous documentation of reversible obstructive airway disease with a positive response to a bronchodilator. Current smokers and patients with a history of $>10$ pack years of smoking were excluded from the study. We also excluded patients with OSA, obesity hypoventilation syndrome, chronic obstructive pulmonary disease, other chronic lung diseases, heart failure, neuromuscular diseases, or other severe chronic systemic illnesses that may affect sleep quality.

Patients were approached by a co-investigator, who explained the purpose of the study. Written informed consent was then obtained from individual or guardian participants, and from the parent for patient who are less than 16 years old. Patient was asked to complete the questionnaires. The questionnaires included seven sections: demographics, Asthma Control Test (ACT), common sleep disorders, risk of OSA (Berlin questionnaire [BQ]), excessive sleepiness (Epworth Sleepiness Scale [EES]), sleep quality (Pittsburgh Sleep Quality Index [PSQI], and insomnia (Insomnia Severity Index [ISI]). All of these questionnaires have been previously validated in Arabic $^{22,24,26,28,30}$

The ACT evaluates the level of asthma control and ranges from 5 (poor control) to 25 (complete control), with higher scores reflecting greater asthma control. An ACT score $>19$ indicates well-controlled asthma ${ }^{19-22}$. The PSQI assesses sleep quality during the past month and contains seven component scales: sleep quality, sleep latency, sleep duration, sleep efficiency, sleep disturbances, use of sleep medication, and daytime dysfunction. Each component is scored from 0 to 3, yielding a global PSQI score ranging from 0 to 21, with higher scores indicating worse sleep quality. A global PSQI score $>5$ has a sensitivity of $89.6 \%$ and specificity of $86.5 \%$ in differentiating good from poor sleepers ${ }^{23,24}$.The ESS measures daytime sleepiness and rates the likelihood of dozing in eight specific situations, with scores from 0 (no chance of dozing) to 3 (high chance of dozing). Total scores range from 0 to 24 , and a score $>10$ on the ESS is indicative of excessive daytime sleepiness $s^{25,26}$. The BQ identifies patients with a high risk for OSA by assessing snoring severity, excessive daytime sleepiness, history of high blood pressure, and history of obesity. The overall risk score (high/low risk) was used in the analysis ${ }^{27,28}$. Lastly, the ISI measures the nature, severity, and impact of insomnia. It assesses severity of sleep onset, sleep maintenance and early morning wakening problems, sleep dissatisfaction, interference of sleep difficulties with daytime functioning, noticeability of sleep problems by others, and distress caused by the sleep difficulties. Total scores range from 0 to 28 , and a cutoff score of $\geq 10$ has an $86.1 \%$ sensitivity and $87.7 \%$ specificity for detecting insomnia cases ${ }^{29,30}$.

Statistical analysis. All analyses were conducted using Statistical Package for Social Sciences (SPSS; v. 25.0). Continuous variables are summarized as means \pm standard deviations (SD) or medians and interquartile range (IQR) for normal and non-normal distributions, respectively. Categorical data are described by frequencies and percentages. The one-way analysis of variance test was used to compare the means of continuous variables, and the chi-square test was used to compare the proportion of sleep disturbance between patients with different levels of asthma control. Potential risk factors causing poor sleep quality in asthma patients, including age, body mass index (BMI), gender, GERD, and risk of sleep apnea, were analyzed using univariate and multivariate analyses. To identify factors that were strongly associated with poor sleep quality, logistic regression analysis was used. A p value $<0.05$ was considered statistically significant.

Ethics approval and consent to participate. The study was approved by the institutional review board of King Abdullah International Medical Research Center (KAIMRC) in Riyadh, Saudi Arabia. All procedures performed in studies involving human participants were in accordance with the ethical standards of the institutional and/or national research committee and with the 1964 Helsinki declaration and its later amendments .Written informed consent was obtained from individual or guardian participants, and from the parent for patient who are less than 16 years old.

\section{Results}

The study was conducted from August 2018 to April 2019. A total of 200 asthma patients participated in the study and filled out the questionnaires, with a response rate of $90 \%$. Baseline patient characteristics included a mean age of $50.6 \pm 17$ years and female predominance (67\%). According to the ACT, 67 (33.5\%) patients had well-controlled asthma, 55 (27.5\%) had partially controlled asthma, and 78 (39\%) had uncontrolled asthma. Comorbid conditions were present in almost half of the patients (Table 1).

When comparing the mean BMI among different levels of asthma control, patients with well-controlled asthma had lower BMI $\left(28.9 \pm 7.4 \mathrm{~kg} / \mathrm{m}^{2}\right)$ compared to the partially controlled group $\left(32.7 \pm 6.8 \mathrm{~kg} / \mathrm{m}^{2}\right)$ and uncontrolled group $\left(32.1 \pm 7.4 \mathrm{~kg} / \mathrm{m}^{2}\right)(\mathrm{p}=0.008)$. 


\begin{tabular}{|c|c|c|c|c|c|}
\hline & \multirow[b]{2}{*}{ All $(N=200)$} & \multicolumn{3}{|l|}{ Asthma control levels } & \multirow[b]{2}{*}{ p value } \\
\hline & & Well-controlled $(\mathrm{N}=67)$ & Partially controlled $(\mathrm{N}=55)$ & Uncontrolled $(\mathrm{N}=78)$ & \\
\hline Age (years) & $50.6 \pm 17.1$ & $48.7 \pm 18.7$ & $52.3 \pm 16.6$ & $51 \pm 16.1$ & 0.49 \\
\hline Female gender & $135(67 \%)$ & $41(61 \%)$ & $37(67 \%)$ & $57(73 \%)$ & 0.31 \\
\hline BMI $\left(\mathrm{kg} / \mathrm{m}^{2}\right)^{\mathrm{a}}$ & $31.2(7.5)$ & $28.9(7.4)$ & $32.7(6.8)$ & $32.1(7.4)$ & 0.008 \\
\hline Never smokers & $193(96 \%)$ & $65(97 \%)$ & $52(94 \%)$ & $76(97 \%)$ & 0.65 \\
\hline Coffee/tea intake & $4(2,6)$ & $4(2,6)$ & $4(2,7)$ & $4(2,7)$ & 0.89 \\
\hline Educated & $176(88 \%)$ & $59(88 \%)$ & $50(91 \%)$ & $67(86 \%)$ & 0.68 \\
\hline Married & $146(73 \%)$ & $47(70 \%)$ & $44(80 \%)$ & $55(71 \%)$ & 0.39 \\
\hline \multicolumn{6}{|l|}{\begin{tabular}{|l|} 
Co-morbidities \\
\end{tabular}} \\
\hline + Allergic rhinitis & $96(48 \%)$ & $35(52 \%)$ & $25(45 \%)$ & $36(46 \%)$ & 0.69 \\
\hline + GERD & $38(19 \%)$ & $15(22 \%)$ & $12(22 \%)$ & $11(14 \%)$ & 0.37 \\
\hline + Hypertension & $90(45 \%)$ & $26(39 \%)$ & $29(53 \%)$ & $35(45 \%)$ & 0.31 \\
\hline + Diabetes mellitus & $71(36 \%)$ & $24(36 \%)$ & $21(38 \%)$ & $26(33 \%)$ & 0.85 \\
\hline \multicolumn{6}{|l|}{ Sleep duration } \\
\hline Less than $6 \mathrm{~h}$ & $95(47 \%)$ & $23(34 \%)$ & $33(60 \%)$ & $39(50 \%)$ & \multirow{2}{*}{0.02} \\
\hline More than $6 \mathrm{~h}$ & $105(53 \%)$ & 44 (66\%) & $22(40 \%)$ & $39(50 \%)$ & \\
\hline
\end{tabular}

Table 1. Baseline characteristics in patients with different levels of asthma control. All other results are reported as means \pm standard deviations or frequencies (percentages). BMI body mass index, GERD

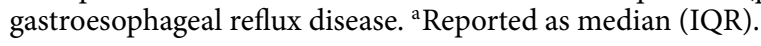

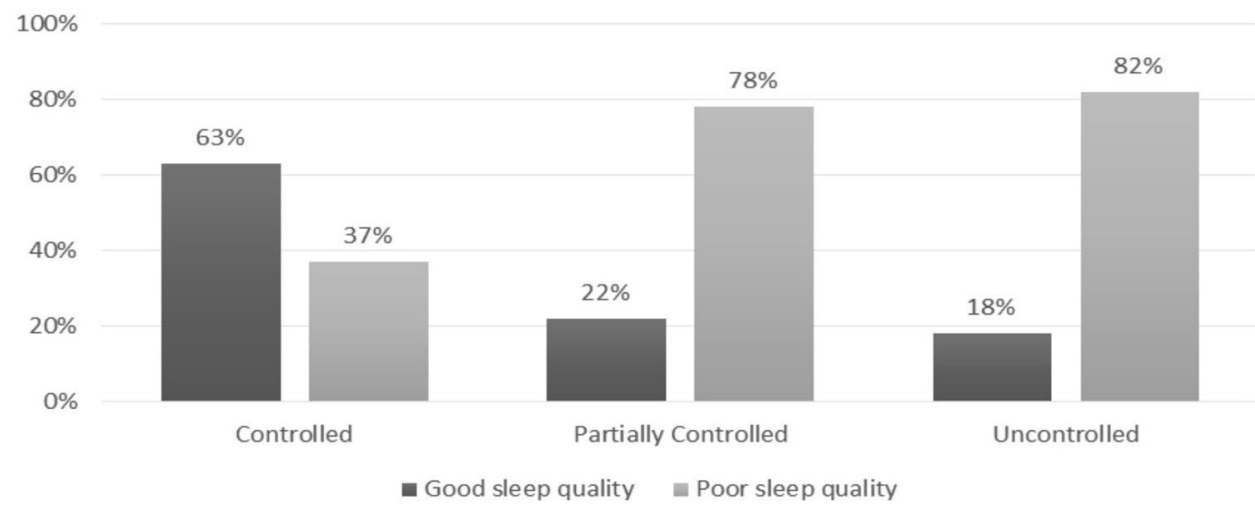

Figure 1. Sleep quality among different level pf asthma control.

The majority of the patients (66\%) suffered from poor sleep quality (PSQI $>5$ ). Almost half of the patients (43\%) had a high risk of OSA, 25\% had excessive daytime sleepiness (ESS > 10), and $46.5 \%$ had significant clinical insomnia (ISI $\geq 10$ ).

Sleep quality and asthma control levels. There was a strong association between sleep quality and asthma control. Poor sleep quality was less common in patients with well-controlled asthma (37\%), compared to those with partially controlled asthma $(78 \%)$ and uncontrolled asthma $(82 \%)(\mathrm{p} \leq 0.001)$ (Fig. 1). Furthermore, patients with well-controlled asthma had less excessive daytime sleepiness, a lower risk of OSA, and lower clinical insomnia compared to the partially controlled and uncontrolled asthma patients $(\mathrm{p}=0.002,0.003$, and $\leq 0.001$, respectively) (Table 2 ).

PSQI domains. The mean global PSQI score was lower in the well-controlled group (5.12 \pm 3.2$)$ than in the partially controlled $(9.07 \pm 3.9)$ and uncontrolled $(9.69 \pm 3.9)$ groups $(\mathrm{p} \leq 0.001)$. In addition, the mean score of all PSQI domains was lower in the well-controlled group compared to the partially controlled and uncontrolled groups, with the exception of sleep duration and use of sleep medication, which were only different between well-controlled and uncontrolled groups (Table 3).

Multivariable analysis. Multivariable analysis was performed to identify factors that were strongly associated with poor sleep quality. The following factors were significantly associated with sleep disturbance: high risk for OSA (odds ratio [OR]: 2.46 [95\% confidence interval $\{\mathrm{CI}\} 1.13,5.35]$ ), partially controlled asthma (OR: 


\begin{tabular}{|c|c|c|c|c|}
\hline & \multicolumn{3}{|c|}{ Asthma control levels } & \multirow[b]{2}{*}{ p value } \\
\hline & $\begin{array}{l}\text { Well-controlled } \\
N=67\end{array}$ & $\begin{array}{l}\text { Partially controlled } \\
\mathrm{N}=55\end{array}$ & $\begin{array}{l}\text { Uncontrolled } \\
\mathrm{N}=78\end{array}$ & \\
\hline PSQI, global score & $5.12 \pm 3.2$ & $9.07 \pm 3.9$ & $9.69 \pm 3.9$ & $<0.001$ \\
\hline Good sleep quality, global PSQI $\leq 5$ & $42(63 \%)$ & $12(22 \%)$ & $14(18 \%)$ & \multirow{2}{*}{$<0.001$} \\
\hline Poor sleep quality, global PSQI > 5 & $25(37 \%)$ & $43(78 \%)$ & $64(82 \%)$ & \\
\hline ESS score & $3.69 \pm 4.06$ & $6.78 \pm 4.2$ & $7.91 \pm 4.85$ & $<0.001$ \\
\hline Excessive daytime sleepiness, ESS $>10$ & $7(10 \%)$ & $15(27 \%)$ & $28(36 \%)$ & 0.002 \\
\hline Berlin Questionnaire score & $0.94 \pm 0.88$ & $1.64 \pm 0.95$ & $1.51 \pm 1.0$ & $<0.001$ \\
\hline Low risk for OSA & $49(73 \%)$ & $24(44 \%)$ & $41(53 \%)$ & \multirow{2}{*}{0.003} \\
\hline High risk for OSA & $18(27 \%)$ & $31(56 \%)$ & $37(47 \%)$ & \\
\hline ISI score & $4.96 \pm 5.75$ & $10.69 \pm 6.73$ & $12.91 \pm 6.52$ & $<0.001$ \\
\hline Insomnia, ISI $\geq 10$ & $13(19 \%)$ & $27(49 \%)$ & $53(68 \%)$ & $<0.001$ \\
\hline
\end{tabular}

Table 2. Sleep quality, daytime sleepiness, and insomnia in patients with different levels of asthma control. PSQI Pittsburgh sleep quality index, ESS Epworth sleepiness scale, OSA obstructive sleep apnea, ISI insomnia severity index.

\begin{tabular}{|c|c|c|c|c|}
\hline \multirow[b]{2}{*}{ PSQI items } & \multicolumn{3}{|c|}{ Asthma control levels } & \multirow[b]{2}{*}{ p value } \\
\hline & $\begin{array}{l}\text { Well-controlled } \\
N=67\end{array}$ & $\begin{array}{l}\text { Partially controlled } \\
\mathrm{N}=55\end{array}$ & $\begin{array}{l}\text { Uncontrolled } \\
\mathrm{N}=78\end{array}$ & \\
\hline PSQI $>5, \mathrm{n}(\%)$ & $25(37 \%)$ & $43(78 \%)$ & $64(82 \%)$ & $<0.001$ \\
\hline Global PSQI (0-21) & $5.12 \pm 3.2$ & $9.07 \pm 3.9$ & $9.69 \pm 3.9$ & $<0.001$ \\
\hline \multicolumn{5}{|l|}{ PSQI domains (0-3) } \\
\hline Subjective sleep quality & $0.7 \pm 0.89$ & $1.29 \pm 0.94$ & $1.69 \pm 0.68$ & $<0.001$ \\
\hline Sleep latency & $0.82 \pm 1.0$ & $1.53 \pm 0.92$ & $1.64 \pm 0.98$ & $<0.001$ \\
\hline Sleep duration & $1.06 \pm 1.14$ & $1.78 \pm 1.23$ & $1.51 \pm 1.26$ & 0.004 \\
\hline Sleep efficiency & $0.61 \pm 0.89$ & $1.45 \pm 1.3$ & $1.21 \pm 1.25$ & $<0.001$ \\
\hline Sleep disturbances & $1.13 \pm 0.63$ & $1.53 \pm 0.6$ & $1.85 \pm 0.58$ & $<0.001$ \\
\hline Use of sleeping medication & $0.07 \pm 0.4$ & $0.22 \pm 0.7$ & $0.38 \pm 0.9$ & 0.035 \\
\hline Daytime dysfunction & $0.72 \pm 0.97$ & $1.27 \pm 1.0$ & $1.41 \pm 0.97$ & $<0.001$ \\
\hline
\end{tabular}

Table 3. Comparison of the Pittsburgh Sleep Quality Index (PSQI) between patients with different levels of asthma control. PSQI Pittsburgh Sleep Quality Index.

\begin{tabular}{|c|c|c|c|c|c|}
\hline & \multicolumn{5}{|c|}{ Association with poor sleep (PSQI score > 5) } \\
\hline & \multirow{2}{*}{\begin{tabular}{|l|} 
Univariate analysis \\
B
\end{tabular}} & \multicolumn{3}{|c|}{ Multivariable analysis } & \multirow[b]{2}{*}{ p-value } \\
\hline & & p-value & $\operatorname{Exp}(B)$ & 95\% CI for $\operatorname{EXP}(B)$ & \\
\hline Age & 0.02 & 0.07 & 1.01 & $(0.99,1.03)$ & 0.25 \\
\hline BMI & 0.05 & 0.02 & 0.99 & $(0.94,1.04)$ & 0.64 \\
\hline Female gender & 0.59 & 0.06 & 1.88 & $(0.88,4.02)$ & 0.10 \\
\hline + Allergic rhinitis & 0.06 & 0.85 & 1.23 & $(0.62,2.45)$ & 0.55 \\
\hline + GERD & -0.70 & 0.056 & 0.47 & $(0.21,1.07)$ & 0.07 \\
\hline High risk for OSA & 1.19 & $<0.001$ & 2.46 & $(1.13,5.35)$ & 0.02 \\
\hline \multicolumn{6}{|l|}{ Asthma control } \\
\hline Partially controlled & 1.80 & $<0.001$ & 5.37 & $(2.25,12.84)$ & $<0.001$ \\
\hline Uncontrolled & 2.04 & $<0.001$ & 6.63 & $(2.98,14.75)$ & $<0.001$ \\
\hline
\end{tabular}

Table 4. Factors associated with sleep quality in asthma patients. $B M I$ body mass index, $C I$ confidence interval, GERD gastroesophageal reflux disease, OSA obstructive sleep apnea, PSQI Pittsburgh Sleep Quality Index. 
5.35 [95\% CI 2.25, 12.84]), and uncontrolled asthma (OR: 6.63 [95\% CI 2.98, 14.75]) (Table 4). No associations between the other covariates and sleep disturbance were found.

\section{Discussion}

This study investigated the prevalence of sleep disturbance among Saudi adult asthmatic patients attending pulmonary clinics at KAMC. Approximately two third (66\%) of the sample study reported having sleep disturbances. This was similar to results from a previous studies that demonstrated a high prevalence of sleep disturbance among patients with asthma $[40-90 \%]^{4-7,31}$. The prevalence of uncontrolled asthma was 39\%. This was in accordance with a previous studies showing that the prevalence of uncontrolled asthma among asthma patients in pulmonary clinics was $40-82 \%$ 7,21,22,32.

Another important finding of this study was that asthma control had an inverse relationship with sleep disturbance. In comparison to patients with partially controlled or uncontrolled asthma, patients with well-controlled asthma were less likely to report poor sleep quality. Similarly, previous studies from different countries have shown that patients with better asthma control have less sleep disturbance ${ }^{4,5,14,16,31,33}$. Our study also demonstrated sleep disturbances in patients with well-controlled asthma (37\%). Braido et al. reported that $10-20 \%$ of totally controlled asthma patients still have poor sleep quality ${ }^{4}$. Luyster et al. reported mean PSQI scores $>5$ in both severe and non-severe asthma patients ${ }^{16}$.

There was no association between gender or age and sleep disturbance. In contrast, Janson et al. reported that the percentage of asthma patients with difficulties maintaining sleep was higher in females (55\%) compared to males $(31 \%)$. The same study showed that age was associated with a decline in estimated sleep duration ${ }^{34}$.

Asthma patients have a higher prevalence of snoring and OSA compared to the general population, and asthma is an established risk factor for OSA ${ }^{5,35}$. In addition, patients with asthma and OSA are seven times more likely to develop severe asthma ${ }^{36}$. Some factors that might play a role in the interaction between asthma and OSA include local inflammation in the upper airway, allergic rhinitis, systemic inflammation, circulating leptin, neuromechanical reflex bronchoconstriction, intermittent hypoxia, GERD, obesity, and asthma therapy ${ }^{37}$. Julien et al. used overnight home polysomnography to investigate the prevalence and severity of OSA in patients with different levels of asthma. The frequency of OSA-hypopnea events was higher in severe asthmatics compared to moderate asthmatics or non-asthmatics ${ }^{14}$. In the current study, $43 \%$ of the asthmatics demonstrated high risk for OSA. Similarly, Lu et al. utilized the STOP-Bang questionnaire to show that $44 \%$ of the asthmatics in their cohort were at high risk for OSA ${ }^{35}$. When adjusting for other covariates, our results show that a high risk for OSA was significantly associated with poor sleep quality.

Using multi-regression analysis, the current study shows that having rhino-sinusitis did not increase the chance of having sleep disturbance in asthmatics. This is similar to findings reported in previous studies ${ }^{4,16}$. Nevertheless, Janson et al. reported that allergic rhinitis is associated with difficulty inducing sleep, daytime tiredness, and daytime sleepiness 5 . In our study, patients with co-morbid GERD did not experience increased sleep disturbance compared to those without GERD. Similarly, Luyster et al. reported that the majority of patients with asthma had poor sleep quality regardless of the presence of GERD ${ }^{16}$.

Excessive daytime sleepiness as a result of sleep disturbance has been previously reported among asthma patients $5,7,18,33$. Our study reveals that $27-36 \%$ of patients with uncontrolled or partially controlled asthma had excessive daytime sleepiness, whereas only $10 \%$ of well-controlled asthma patients had excessive daytime sleepiness. Campos et al. reported significantly increased daytime sleepiness with worse asthma control among 123 women with asthma ${ }^{33}$. Teodorescu et al. also reported a significantly higher percentage of excessive daytime sleepiness in patients with severe asthma (31\%), compared to those with non-severe asthma $(19 \%)^{18}$.

The current study also looked at the relationship between insomnia and asthma control. Insomnia was identified in $68 \%$ of patients with uncontrolled asthma, $49 \%$ with partially controlled asthma, and $19 \%$ with well-controlled asthma. Luyster et al. reported 37\% of patients suffering from insomnia among 714 patients with physician-diagnosed asthma, and insomnia was significantly associated with worse asthma control, worse quality of life, and increased depression ${ }^{17}$. Another study showed that the prevalence of insomnia symptoms was common among patients with asthma and asthma-chronic obstructive pulmonary disease overlap (ACO), in comparison to patients without airway diseases ${ }^{38}$.

The current study had several limitations. First, the sample size was small, and it was predominantly female. Second, the study was performed in a single tertiary referral center, which may have introduced referral bias and may not accurately represent the prevalence of the sleep disturbance among asthma patients in the community or in other geographical areas. Third, the cross-sectional design prevented against inferences regarding the directionality of the relationship between sleep quality and asthma control. Lastly, accurate measurements of sleep disturbance, such as sleep studies for OSA and sleep diaries, were not performed.

\section{Conclusions}

Poor sleep quality was common among patients with asthma, especially those with suboptimal levels of asthma control. In addition, patients with uncontrolled asthma had a higher risk for OSA and were more likely to report insomnia and excessive daytime sleepiness. Further studies are needed to better understand the interaction between asthma and sleep.

\section{Data availability}

The datasets used and/or analysed during the current study are available from the corresponding author on reasonable request. 
Received: 8 May 2020; Accepted: 11 December 2020

Published online: 28 January 2021

\section{References}

1. National Asthma Education and Prevention Program: Expert panel report III: Guidelines for the diagnosis and management of asthma. Bethesda, MD: National Heart, Lung, and Blood Institute, 2007. (NIH publication no. 08-4051) (Accessed 03 June 2019). www.nhlbi.nih.gov/guidelines/asthma/asthgdln.htm.

2. Global Initiative for Asthma. Pocket Guide for asthma management and prevention for adults and children older than 5 years (accessed 03 June 2019). https://ginasthma.org/archived-reports/ (2018).

3. Mohamed Hussain, S., Ayesha Farhana, S. \& Mohammed, A. S. Time trends and regional variation in prevalence of asthma and associated factors in Saudi Arabia: A systematic review and meta-analysis. Biomed. Res. Int. 2018, 8102527 (2018).

4. Braido, F. et al. Sleep disturbances and asthma control: A real life study. Asian Pac. J. Allergy Immunol. 27, 27-33 (2008) (PubMed: 19548627).

5. Janson, C. et al. Increased prevalence of sleep disturbances and daytime sleepiness in subjects with bronchial asthma: A population study of young adults in three European countries. Eur. Respir. J. 9, 2132-2138 (1996) (PubMed: 8902479).

6. Krouse, H. J., Yarandi, H., McIntosh, J., Cowen, C. \& Selim, V. Assessing sleep quality and daytime wakefulness in asthma using wrist actigraphy. J. Asthma. 45, 389-395 (2008) (PubMed: 18569232).

7. Mastronarde, J. G., Wise, R. A., Shade, D. M., Olopade, C. O. \& Scharf, S. M. Sleep quality in asthma: Results of a large prospective clinical trial. J. Asthma. 45, 183-189 (2008) (PubMed: 18415823).

8. Kavanagh, J., Jackson, D. J. \& Kent, B. D. Sleep and asthma. Curr. Opin. Pulm. Med. 24(6), 569-573 (2018).

9. Bonnet, R., Jorres, R., Heitmann, U. \& Magnussen, H. Circadian rhythm in airway responsiveness and airway tone in patients with mild asthma. J. Appl. Physiol. 71, 1598-1605 (1991).

10. Martin, R. J., Cicutto, L. C. \& Ballard, R. D. Factors related to the nocturnal worsening of asthma. Am. Rev. Respir. Dis. 141, 33-38 (1990).

11. Alt, J. A. et al. Sleep quality outcomes after medical and surgical management of chronic rhinosinusitis. Int. Forum Allergy Rhinol. 7, 113-118 (2017).

12. Davies, S. E. et al. The association between asthma and obstructive sleep apnea (OSA): A systematic review. J. Asthma 56(2), $118-129$ (2019).

13. Tsuno, N., Besset, A. \& Ritchie, K. Sleep and depression. J. Clin. Psychiatry 66, 1254-1269 (2005).

14. Julien, J. Y. et al. Prevalence of obstructive sleep apnea-hypopnea in severe versus moderate asthma. J. Allergy Clin. Immunol. 124(2), 371-376 (2009).

15. Kong, D.-L. et al. Association of obstructive sleep apnea with asthma: A meta-analysis. Sci. Rep. 7, 4088 (2017).

16. Luyster, F. S. et al. Sleep quality and asthma control and quality of life in nonsevere and severe asthma. Sleep Breath. 16(4), 1129-1137 (2012).

17. Luyster, F. S. et al. Association between insomnia and asthma burden in the Severe Asthma Research Program (SARP) III. Chest 150(6), 1242-1250 (2016).

18. Teodorescu, M. et al. Obstructive sleep apnea risk, asthma burden, and lower airway inflammation in adults in the Severe Asthma Research Program (SARP) II. J. Allergy Clin. Immunol. Pract. 3, 566-575 (2015) (e1).

19. Nathan, R. A. et al. Development of the asthma control test: a survey for assessing asthma control. J. Allergy Clin. Immunol. 113(1), 59-65 (2004).

20. Schatz, M. et al. Asthma Control Test: Reliability, validity, and responsiveness in patients not previously followed by asthma specialists. J. Allergy Clin. Immunol. 117(3), 549-556 (2006).

21. Al-Zahrani, J. M. et al. Factors associated with poor asthma control in the outpatient clinic setting. Ann. Thorac. Med. 10(2), 100-104 (2015).

22. Al-Jahdali, H. H., Al-Hajjaj, M. S., Alanezi, M. O., Zeitoni, M. O. \& Al-Tasan, T. H. Asthma control assessment using asthma control test among patients attending 5 tertiary care hospitals in Saudi Arabia. Saudi Med. J. 29, 714-717 (2008).

23. Buysse, D. J., Reynolds, C. F., Monk, T. H., Berman, S. R. \& Kupfer, D. J. The Pittsburgh Sleep Quality Index: A new instrument for psychiatric practice and research. Psychiatry Res. 28(2), 193-213 (1989).

24. Suleiman, K. H., Yates, B. C., Berger, A. M., Pozehl, B. \& Meza, J. Translating the pittsburgh sleep quality index into Arabic. West J. Nurs Res 32(2), 250-268 (2010).

25. Johns, M.W. A new method for measuring daytime sleepiness: The Epworth Sleepiness Scale. Sleep 50-55 (1991).

26. Ahmed, A. E. et al. Validation of the Arabic version of the Epworth Sleepiness Scale. J. Epidemiol. Glob. Health 4(4), 297-302 (2014).

27. Netzer, N. C., Stoohs, R. A., Netzer, C. M., Clark, K. \& Strohl, K. P. Using the Berlin Questionnaire to identify patients at risk for the sleep apnea syndrome. Ann. Intern. Med. 131(7), 485-491 (1999).

28. Saleh, A. B., Ahmad, M. A. \& Awadalla, N. J. Development of Arabic version of Berlin questionnaire to identify obstructive sleep apnea at risk patients. Ann. Thorac. Med. 6(4), 212-216 (2011).

29. Morin, C. M. Insomnia, Psychological Assessment and Management (Guilford Press, New York, 1993).

30. Suleiman, K. H. \& Yates, B. C. Translating the insomnia severity index into Arabic. J. Nurs. Scholarship 43, 49-53 (2011).

31. Vukoja, M. et al. Sleep quality and daytime sleepiness in patients with COPD and asthma. Clin. Respir. J. 12(2), 398-403 (2018).

32. Marcus, P. et al. CHARIOT Study Investigators. A retrospective randomized study of asthma control in the US: Results of the CHARIOT study. Curr. Med. Res. Opin. 24, 3443-3452 (2008).

33. Campos, F. L. et al. Depressive symptoms, quality of sleep, and disease control in women with asthma. Sleep Breath 21(2), 361-367 (2017).

34. Janson, C., Gislason, T., Boman, G., Hetta, J. \& Roos, B. E. Sleep disturbances in patients with asthma. Respir. Med. 84(1), 37-42 (1990).

35. Lu, H. et al. Screening for obstructive sleep apnea syndrome in asthma patients: A prospective study based on Berlin and STOPBang questionnaires. J. Thorac. Dis. 9(7), 1945-1958 (2017).

36. Teodorescu, M. et al. Asthma control and its relationship with obstructive sleep apnea (OSA) in older adults. Sleep Disord. 2013, 251567 (2013).

37. Sherbini, N., Al-Harbi, A., Khan, M. \& Al-Jahdali, H. Asthma and sleep. In Synopsis of Sleep Medicine [Book on the Internet] 1st edn (ed. Pandi-Perumal, S. R.) 363-377 (Apple Academic Press, Oakville, 2016).

38. Mindus, S. et al. Asthma and COPD overlap (ACO) is related to a high burden of sleep disturbance and respiratory symptoms: Results from the RHINE and Swedish GA2LEN surveys. PLoS ONE 13(4), e0195055 (2018).

\section{Author contributions}

All authors made substantial contributions to conception and design, acquisition of data or analysis and interpretation of data; took part in drafting the article or revising it critically for important intellectual content; gave final approval of the version to be published. 


\section{Competing interests}

The authors declare no competing interests.

\section{Additional information}

Correspondence and requests for materials should be addressed to A.A.-H.

Reprints and permissions information is available at www.nature.com/reprints.

Publisher's note Springer Nature remains neutral with regard to jurisdictional claims in published maps and institutional affiliations.

(c) (i) Open Access This article is licensed under a Creative Commons Attribution 4.0 International License, which permits use, sharing, adaptation, distribution and reproduction in any medium or format, as long as you give appropriate credit to the original author(s) and the source, provide a link to the Creative Commons licence, and indicate if changes were made. The images or other third party material in this article are included in the article's Creative Commons licence, unless indicated otherwise in a credit line to the material. If material is not included in the article's Creative Commons licence and your intended use is not permitted by statutory regulation or exceeds the permitted use, you will need to obtain permission directly from the copyright holder. To view a copy of this licence, visit http://creativecommons.org/licenses/by/4.0/.

(c) The Author(s) 2021 